\title{
Regulatory radiation risks for the population and natural objects within the semipalatinsk test site
}

\author{
S.I. Spiridonov ${ }^{1}$, V.L. Tetenkin ${ }^{1}$, M.K. Mukusheva ${ }^{2}$ and I.E. Epifanova ${ }^{1}$ \\ ${ }^{1}$ Russian Institute of Agricultural Radiology and Agroecology, 249032 Obninsk, Russia \\ ${ }^{2}$ National Nuclear Center of Kazakhstan, 071100 Kurchatov, Kazakhstan
}

\begin{abstract}
Advisability of using risks as indicators for estimating radiation impacts on environmental objects and humans has been justified. Results are presented from identification of dose burdens distribution to various cohorts of the population living within the Semipalatinsk Test Site (STS) and consuming contaminated farm products. Parameters of dose burden distributions are estimated for areas of livestock grazing and the most contaminated sectors within these areas. Dose distributions to meadow plants for the above areas have been found. Regulatory radiation risks for the STS population and meadow ecosystem components have been calculated. Based on the parameters estimated, levels of radiation exposure of the population and herbaceous plants have been compared.
\end{abstract}

\section{INTRODUCTION}

Farming within the Semipalatinsk Test Site (STS) under radioactive contamination generates a need to analyze the radiation impacts for the STS population [1]. Based on a system of models describing ${ }^{90} \mathrm{Sr}$ and ${ }^{137} \mathrm{Cs}$ behavior in the STS meadow systems, screening estimations were carried out of the accumulation of these radionuclides in farm products obtained within the STS. The results have demonstrated that when horses and sheep graze the most contaminated industrial sites "Balapan" and "Ground Zero", the concentration of the long-lived radionuclides in the farm products may exceed the limits adopted in Kazakhstan [2]. A logical extension of works on the assessment of the STS radioecological situation is dose estimation to the population and risk assessment. Components of pasture ecosystems are permanently exposed to ionizing radiation formed by long-lived radionuclides. In this context, of great interest is estimation of radiation effects not only on humans but also on the main component of these ecosystems - herbaceous plants.

The objectives of the study, the results of which are summarized in the present paper, were:

- elaboration of a method for assessing consequences of radioactive contamination of ecosystems using radiation risks as unified indicators;

- estimation of radiation risks for the STS population and the main component of meadow ecosystems herbaceous vegetation.

\section{METHOD OF RISK ESTIMATION}

Radiation (radioecological) risks, assessed for the population and biota components within the Semipalatinsk Test Site, are a probabilistic characteristic. The choice of the risk assessment method is primarily dictated by the level of information support on parameters necessary for the computation. Among these parameters are: characteristic of the acting factor (dose burden) and sensitivity of the study object to this factor. To formalize the latter, risk criteria, whose choice is specified by peculiar features of the objects considered and the aims of the study, are justified. 
The most adequate, in terms of the description depth of the radiation impact and at the same time "information consuming", is a stochastic approach to risk assessment. As input data, this approach makes use of statistical distributions of both acting factor characteristic and risk assessment criterion. When this information is available, risk is estimated based on the product of probabilities characterizing statistical distributions of the factor and criterion. The implementation of a simpler semi-stochastic approach is feasible with deterministic (point) values of risk criteria. Identification of these criteria is, however, a heavy task where substantial subjectivity is difficult to avoid.

Classified as semi-stochastic are regulatory risks. These are calculated based on the adopted and recommended limits of the radiation factor effects. A permissible level of extra irradiation of the population ( $1 \mathrm{mSv} /$ year) may be used as this limit [3]. Worth noting that currently no legal international standards are available which limit ionizing radiation effects on biota. Therefore, as threshold values of the dose burden in calculations, a number of standards for terrestrial plants cited in the literature were used (400 and $100 \mu \mathrm{Gy} / \mathrm{h}$ ) [4, 5]. Besides, as a more stringent criterion, a screening dose rate limit of $10 \mu \mathrm{Gy} / \mathrm{h}$ was considered [6].

The regulatory risks for various groups of the population and meadow vegetation exposed to irradiation as a result of STS area contamination were calculated as follows:

$$
\text { Risk }=\int_{D N}^{\infty} P(D) d D,
$$

where $P(D)$ is the frequency distribution of dose burden to the population when agricultural animals are pastured within the established grazing areas, or to the meadow vegetation growing in these areas; $D N$ is the dose limit for the population or the component of the environment studied.

Two cohorts of the population simultaneously exposed to external and internal irradiation have been identified: herdsmen of farm animals and the remaining population within the STS area. The third category included the residents outside the STS area who received additional irradiation only from the consumption of products contaminated by radionuclides. Radiation risks to the population and meadow plants have been assessed for the areas of livestock (horses and sheep) grazing in the vicinity of technical sites "Ground Zero" and "Balapan". At these sites there are pastures of the collective farms, such as "Chaganskoe" (wintering ground "Atomic Lake") and "Akzharskoe" (wintering ground "Taktakoil"). Within the conservative assessment scope, the most contaminated sectors in the grazing areas have been identified.

\section{SELECTED RESULTS}

Within the bounds of the radiation risk assessment, the dose burden distributions have been identified for various population cohorts for the situation when animals were grazing on four types of pastures contaminated after the nuclear weapons tests within the Semipalatinsk Test Site. Doses of internal and external exposure of the population were estimated using dosimetric models parameterized for the STS conditions and experimental data describing the contamination densities by long-lived radionuclides of the grazing areas and most affected sectors [7]. As a result of computations, empirical frequencies have been derived that describe discrete distributions of dose burdens to all study cohorts of the population for the investigated territory.

Identification of the continuous distributions of dose burdens was performed using superposition of two cumulative functions of gamma-distribution:

$$
F(D)=\int_{0}^{D}\left[\sum_{i} C_{i} \cdot G_{i}(x)\right] d x
$$


where $D$ is the dose rate value, $G_{i}(x)$ is the probability density of the $i$-th gamma-distribution, $C_{i}$ is the weighting factor.

The equation for the probability density of gamma-distribution takes the form:

$$
G_{\alpha, m}(x)=\frac{\alpha}{m \Gamma(\alpha)}\left(x \frac{\alpha}{m}\right)^{\alpha-1} \exp \left(-x \frac{\alpha}{m}\right)
$$

where $m$ is the random value average, $\alpha$ is the form parameter $\left(\alpha=m^{2} / \mathrm{s}^{2}\right), s^{2}$ is the random value variance, $\Gamma(\alpha)$ is the Euler gamma-function.

The comparison of cumulative histograms and continuous functions of dose burden distribution to herdsmen staying on pastures all year round is illustrated in Fig. 1. Superposition of gamma-functions adequately describes a complex pattern of radioactive contamination as a result of imposition of traces from nuclear explosions. Fig. 2 illustrates the probability density function of dose burdens to different cohorts of the STS population consuming contaminated products (for the grazing areas centered at the wintering grounds "Taktakoil" and "Atomic Lake" and for the most affected sectors).

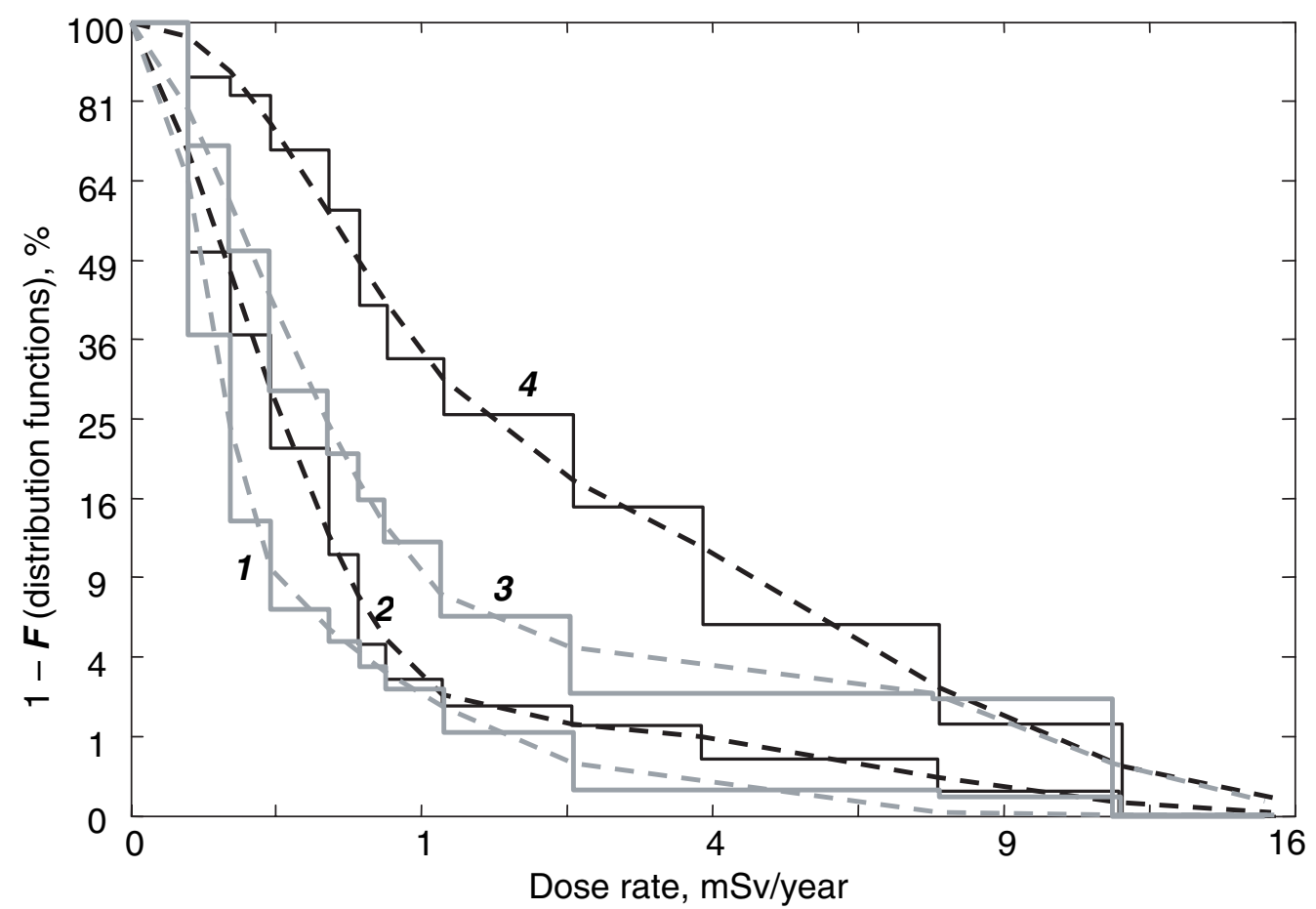

Figure 1. Comparison of estimated distribution functions and experimental histograms describing dose burdens to herdsmen spending the whole year on pastures: 1 and 3 - "Taktakoil" (grazing area and contaminated sector); 2 and 4 - "Atomic lake" (grazing area and contaminated sector). Solid lines - experimental data, dotted lines gamma-distributions.

The regulatory risks defining probabilities of dose limit exceeding ( $1 \mathrm{mSv} / \mathrm{year})$ for different population cohorts and 4 types of pasture are given in Table 1. Analysis of the derived information allows a conclusion that when horses and sheep graze the most contaminated plots adjacent to "Atomic Lake", the probabilities of dose limit exceeding range from 0.11 for the population which do not take part in animal pasturing to 0.34 for herdsmen working all year round. Risks estimated for grazing conditions within other areas range between $7 \cdot 10^{-4}$ and $9.3 \cdot 10^{-2}$. Significant risk values for the area adjacent to 
the "Atomic Lake" formed after an excavated nuclear explosion result from the fact that near this lake there are plots with rather high densities of radioactive contamination [8].

To compare different approaches to estimating radiation factor effects on the STS population, indices of radiation exposure, RIF [9], have been calculated, which are ratios of dose burdens estimated on the basis of mean weighted densities of the study areas contamination to the critical dose of irradiation ( $1 \mathrm{mSv} /$ year). RIF values for animal grazing within the most contaminated sector range from 0.37 (for the population consuming contaminated products) to 1.47 (for herdsmen working the whole year). The comparison of indices of radiation exposure and regulatory "semi-stochastic" risks (Table 1) shows that these parameters are interrelated non-lineally. This results from the fact that indices are estimated ignoring dose burden distributions, which vary considerably depending on the radioecological characteristics of specific territories.

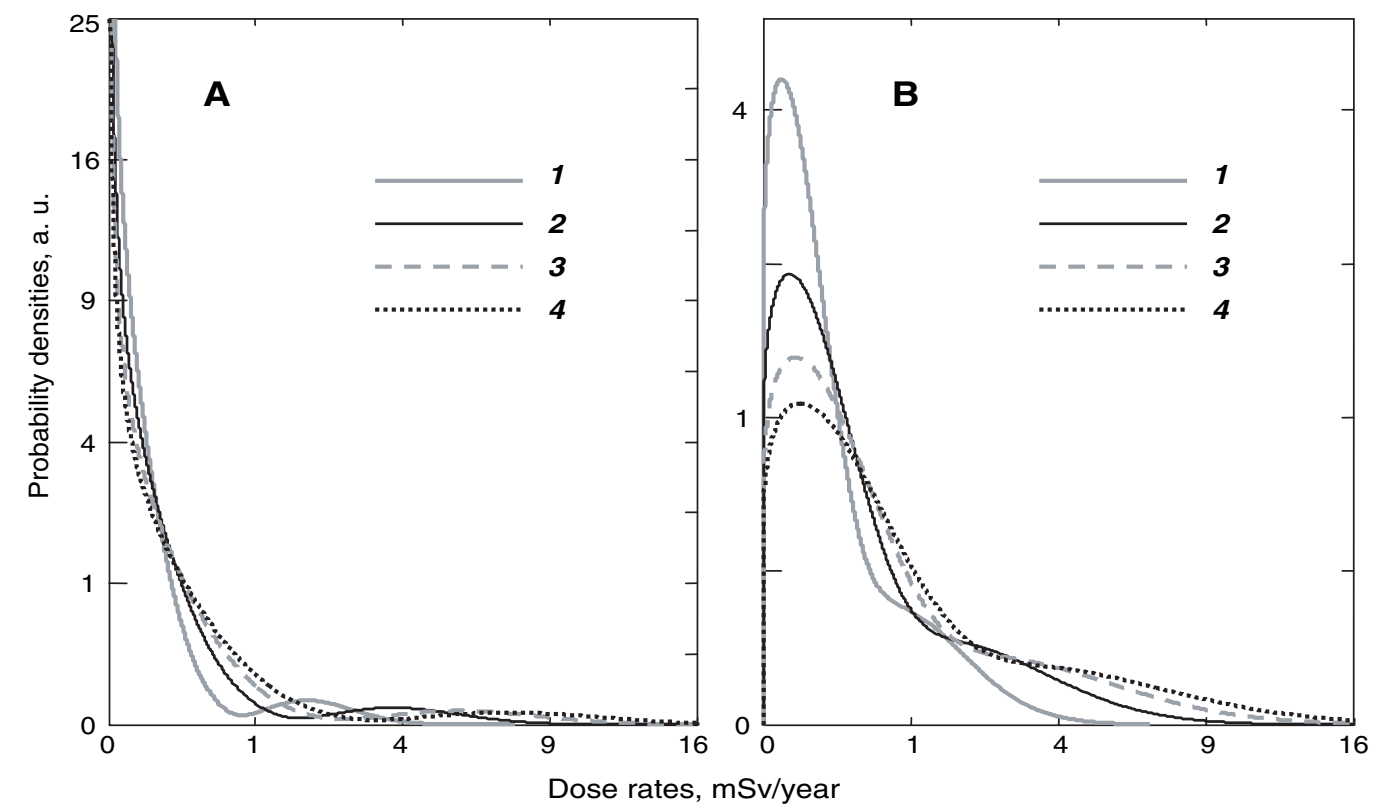

Figure 2. Probability density function of dose burdens to different population cohorts within STS for the situation of animal grazing on contaminated sectors of the "Taktakoil" (A) and "Atomic lake" (B) pastures. 1 and 2 - the population outside and within STS area; 3 and 4 -herdsmen who are absent and present on pasture in the wintertime.

Table 1. Probabilities of dose limit (1 mSv/year) exceeding for different population cohorts living within STS and consuming contaminated products.

\begin{tabular}{|l|c|c|c|c|}
\hline Area of farm animals grazing & $\begin{array}{c}\text { Population } \\
\text { living outside } \\
\text { STS }\end{array}$ & $\begin{array}{c}\text { STS } \\
\text { population }\end{array}$ & $\begin{array}{c}\text { Herdsmen } \\
\text { (year-round } \\
\text { grazing) }\end{array}$ & $\begin{array}{c}\text { Herdsmen } \\
\text { (excluding } \\
\text { winter period) }\end{array}$ \\
\hline "Taktakoil", grazing area & $7.0 \cdot 10^{-4}$ & $6.7 \cdot 10^{-3}$ & $2.3 \cdot 10^{-2}$ & $1.5 \cdot 10^{-2}$ \\
\hline "Atomic lake", grazing area & $9.3 \cdot 10^{-3}$ & $1.4 \cdot 10^{-2}$ & $3.0 \cdot 10^{-2}$ & $1.9 \cdot 10^{-2}$ \\
\hline "Taktakoil", most affected sector & $4.2 \cdot 10^{-2}$ & $4.7 \cdot 10^{-2}$ & $9.3 \cdot 10^{-2}$ & $6.6 \cdot 10^{-2}$ \\
\hline "Atomic lake", most affected sector & 0.11 & 0.20 & 0.34 & 0.27 \\
\hline
\end{tabular}

Dose burdens to STS meadow vegetation were estimated for the pastures adjacent to the sites "Ground Zero" (wintering ground "Taktakoil") and "Balapan" (wintering ground "Atomic Lake"). To this end, dosimetric models based on the described approaches [10] were employed. The dosimetric 
models were parameterized using the information submitted by the National Nuclear Center of Kazakhstan. For mathematical description of dose distribution to plants, superposition of two functions of gamma-distribution was used (Equation (2)). Results from processing of spatial heterogeneity of dose burdens to meadow plants are presented in Fig. 3.

Regulatory radiation risks have been estimated (Equation (1)) for the meadow vegetation within the STS pastures lands considered. The values of the radiation risks for meadow vegetation calculated with the use of 100 and $400 \mu \mathrm{Gy} / \mathrm{h}$ as the limits are negligible. With the most severe criterion $(10 \mu \mathrm{Gy} / \mathrm{h})$, the values for the regulatory risks vary between $2.0 \cdot 10^{-6}$ and $8.2 \cdot 10^{-4}$. The highest risk levels for meadow vegetation are observed in the "Atomic Lake" region. For the contaminated sector of the area adjacent to the "Atomic Lake" this value is $8.2 \cdot 10^{-4}$.

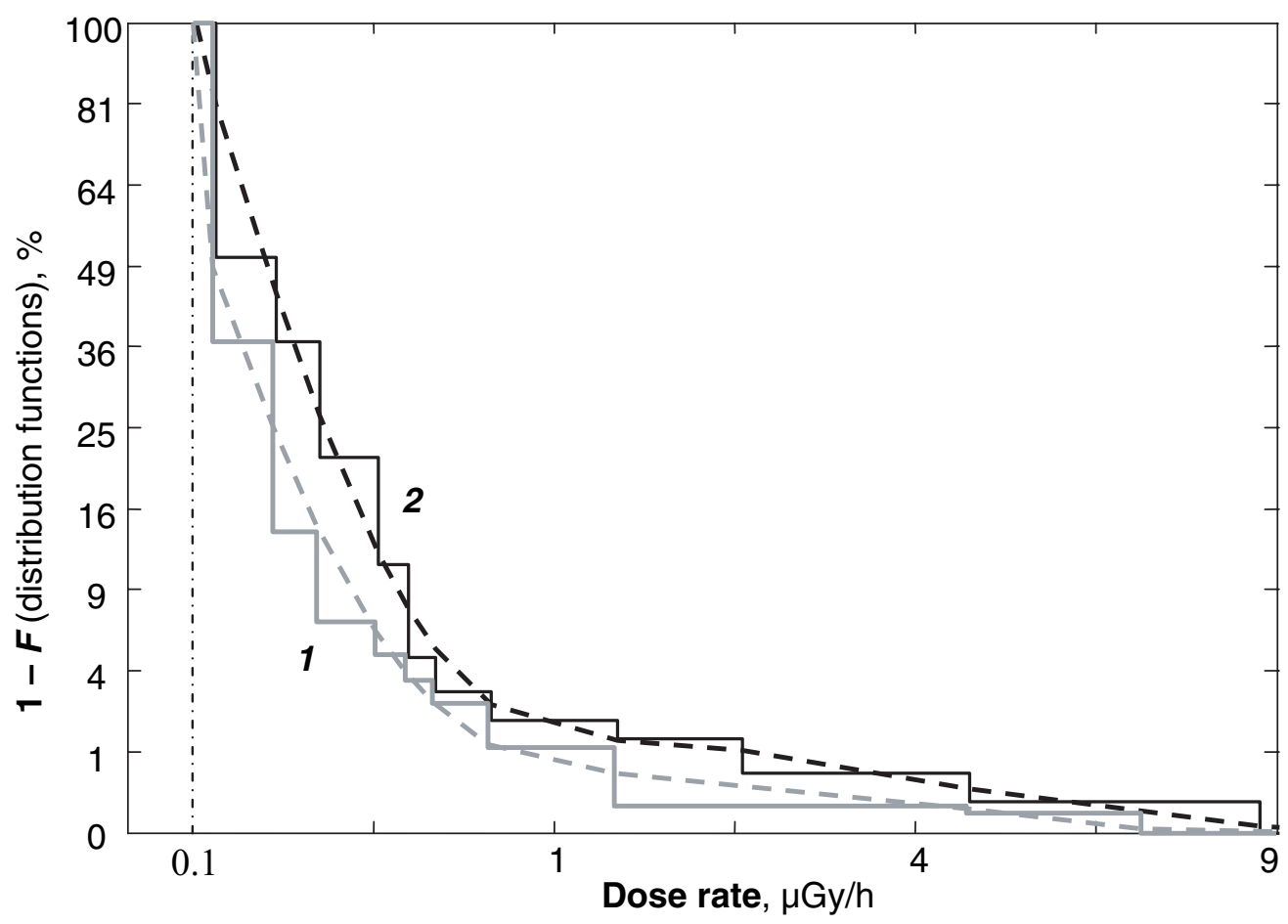

Figure 3. Comparison of estimated distribution functions and experimental histograms describing dose burdens to meadow plants: 1 - "Taktakoil"; 2 - "Atomic Lake". Solid lines - experimental data, dotted lines - gammadistributions.

Based on the regulatory risk assessments for cenosis-generating component of STS meadow ecosystems using literature dose limits for terrestrial plants, it is concluded that ionizing radiation does not produce significant effects on the STS meadow vegetation. At the same time, on small STS plots with high densities of radioactive contamination, there may be effects at cellular and even organism levels of biological organization. To illustrate, mention should be made of a plot in the close proximity to one of the holes where an underground nuclear test has been performed ("Balapan") [11]. Dose rate to herbaceous plants amounts here to $5.7 \mathrm{mGy} /$ day. According to the reported data [12], at dose rates above 4.2-6.3 mGy/day, herbaceous plants can develop morphological alterations. However, small sizes of the plot (several tens meters) make it, probably, impossible to conclude on significant abnormalities of the plant population within large areas involved in the risk assessment. 


\section{CONCLUSIONS}

1. Applicability of a regulatory risk computation procedure has been demonstrated in the framework of a comprehensive analysis of ecosystem radioactive contamination impacts on the public and biota components. Radiation risks, which can be classified in this case as radioecological, are unified indicators applicable for various comparative estimations. Comparison of the regulatory risk values for different population cohorts with those of radiation exposure indices for the same cohorts demonstrates that the relation between these characteristics is non-linear. Since risk assessments take into account dose burden distributions varying in accordance with the peculiar features of the territories considered, the use of probabilistic values for radioecological assessments seems more advantageous.

2. It has been found that the risk to exceed dose limit $(1 \mathrm{mSv} / \mathrm{year})$, when obtaining agricultural products on the most contaminated plot adjoining the "Atomic Lake", reaches a significant value for the critical cohort of the population involved in animal pasturing. It is, therefore, advisable that restrictions be imposed on the use of the most affected plot within testing area "Balapan" for production of agricultural stuffs.

3. Results from the regulatory radiation risk assessments for the STS meadow ecosystems using a set of dose standards have shown that ionizing radiation does not have negative effects on the meadow vegetation widespread within the large-scale areas. On small plots of the STS with high densities of radioactive contamination effects are possible at the lower levels of biological organization. From this point of view, challenging is estimation of genetic impacts. To solve this problem, long-term observations are needed of the development of living communities on the most affected STS plots.

4. Comparison of the radiation exposure levels to humans and herbaceous vegetation has revealed that currently the STS population (particularly its critical group) is exposed to ionizing radiation to a larger extent than herbaceous plants. This conclusion confirms the main postulate of the anthropocentric ICRP concept: "if radiation standards protect man than, then biota are also adequately protected from ionizing radiation in the same situation" [3] with respect to the cenosis-generating component of the STS meadow ecosystems. A similar inference was made from the analysis of radioecological consequences for the long term after the Chernobyl accident [9].

5. For a detailed evaluation of the radiation factor effect on biota components, it is expedient to apply a stochastic approach to risk assessment, which takes into account uncertainties of both acting factor and characteristics of the object considered. In this case, the calculations need statistical distributions of dose burdens and risk criteria. This approach is applicable for a comparative evaluation of the ecosystem impacts of ionizing radiation and factors of non-radiation nature.

\section{References}

[1] Semiochkina N., Voigt G., Mukusheva M. et al., Health Physics 86 (2004).

[2] Spiridonov S.I., Gontarenko I.A.., Mukusheva M.K. et al., Radiation Biology. Radioecology 45 (2005).

[3] Alexakhin R.İ., Fesenko S.V., Radiation Biology. Radioecology 44 (2004).

[4] DOE Standard. A Graded Approach for Evaluation Radiation Doses to Aquatic and Terrestrial Biota. Washington: US Department of Energy, D.C. 20585, Project number ENVR-0011. US Department of Energy (2000).

[5] Bird G., Thompson P., MacDonald C. et al., Supporting Document for the Priority Substances List Assessment of Release of Radionuclides from Nuclear Facilities (Impact on Non-Human Biota). Ottawa: Canadian Nuclear Safety Commission (2000).

[6] D-ERICA: An integrated approach to the assessment and management of environmental risks from ionizing radiation. Project number FI6R-CT-2004-508847. Swedish Radiation Protection Authority (2007). 
[7] Spiridonov S.I., Mukusheva M.K. Shubina O.A. et al., Radiation Biology. Radioecology 48 (2008).

[8] Ptitskaya L.D., Vestnik NNCK 3 (2002).

[9] Fesenko S.V., Alexakhin R.M., Geraskin S.A. et al., Journal of Environmental Radioactivity 80 (2005).

[10] Fesenko S.V., Radiation factor effects on the ecosystem of a fresh water body. Candidate thesis (physics and mathematics). M: MIFI (1985).

[11] USSR nuclear tests: modern radioecological situation at the test sites/Team of authors headed by prof. V.A. Logachev. M.: IzdAT (2002).

[12] Geraskin S.A., Fesenko S.V. and Alexakhin R.M., Environment International 34 (2008). 
